\title{
Wind Power Business Day-ahead Market Strategy Bidding Considering Allowable Deviation and Wind Speed Distribution
}

\author{
Yi Song ${ }^{1, a}$, Xiangyu Zhang ${ }^{2, b}$, Dan Li ${ }^{2}$, Zhengyu Xue ${ }^{1}$, Zhongfu Tan², \\ Zhengdong $\mathrm{Du}^{3}$ \\ ${ }^{1}$ State Power Economic Research Institute, 102209, China \\ ${ }^{2}$ North China Electric Power University, 102206, China \\ ${ }^{3}$ State Grid Zhejiang Electric Power Company Economic Research Institute, 310008, China \\ ahdzxy114@163.com, b469099082@qq.com
}

Keywords: Wind farm business; Day-ahead market; Bidding strategy; Allowable deviation

\begin{abstract}
With the development of large-scale wind power integration and electricity markets, wind farms business were asked to participate in the electricity market. Because of the volatility and unpredictability of wind power, the wind farm suppliers contribute to obtain benefits in the bidding at the same time, need to bear the imbalances cost of inaccurately reporting output resulting. This paper follows the wind itself the objective law, proposed to charge the imbalance cost of the need to consider the power tolerance, detailed derivation of wind farm business day-ahead market bidding model in the case of wind farm wind speed meet Weibull distribution, the model with the wind farm business expected revenue largest as the goal, day-ahead market the wind farm business obtain bidding output revenue, wind farms business charge imbalance costs of more than the allowable deviation of the power section in the imbalance market. The example detailed analysis of the different wind speed distribution parameters and tolerances ratio on the wind farm business's effect of day-ahead market bidding output.
\end{abstract}

\section{Introduction}

By the end of 2013, global wind power installed capacity has reached 318.137 million kilowatts[1]. 2013, China's total installed wind turbines reached 63,120 units, installed capacity reach 91412.89MW. The installed capacity of new and cumulative is the first in the world [2]. Currently, in order to encourage the continued development of wind power, power grid take effective output of full acquisition wind power. But with the increase of wind power levels, due to the wind power characteristics of volatility, inaccurate predictability and intermittent, in order to full acceptance of wind power, power grid pay a large economic cost: the need for more spinning reserve, peaking cost and AGC cost [3]. These increase burdens of conventional power supply and power grid ancillary services. Therefore, power system at the same time encourage the development of wind power, also proposed to allow the wind farms business and conventional energy investors participate in the market at the same time, so wind power bear some power imbalances costs [4].

Because of the wind farm providers need to bear the cost of certain imbalances, leading to some loss of wind farm's income. According to the United Kingdom, Spain and other countries wind power market statistics, revenue loss will reach $10 \%$ of total revenues [5]. Wind farm business in order to obtain the maximum benefit, need to make decisions policy on the day-ahead market declaration for output, making its total earnings maximize in the balance market and day-ahead market. In order to determine the policy of wind power business in the day-ahead market declaration for output, literature [6] considered the characteristics of wind power prediction accuracy over time scales shorter and improve, designed the wind power within-day market, and in the within-day market updated once output of day-ahead market declaration, contributed to raise the wind farm's income, reduced the forecast inaccurate brought about the loss of power imbalance charges. Wind farm business seeks to maximize their own income at the same time in the electricity market, must also bear the risk due to the uncertainty of wind power brings. How the participant is willing to assume the risks and benefits combine assessment is a concern content of wind farm 
business policy declaration output.

Above literature consider wind power deviations cost caused by the characteristic of random and incomplete controllability. Because of this difference in nature, forming a objective fact that wind speed or wind power is difficult to accurately predict that the bias is always present. For improving the prediction accuracy by Continuous improvement measures to improve forecasting and other means and still can't accurately predict that part of the deviation should be grid allowed and accepted, when the deviation of day-ahead market declaration output and the actual output of wind farm is within the allowable range and should not be counted wind farm's imbalance cost. Only power deviation of exceeded the permitted scope only charge imbalance charges. Based on this concept, paper researched bidding strategies of wind farm business in the day-ahead market.

\section{Wind power and electricity market}

\section{Research on wind farm output}

Wind speed is a random variable, by long-term statistics for a large number of measured data show that random distribution of wind speed approximately obey the Weibull distribution function. Its probability density function:

$$
\varphi(v)=\frac{k}{c}\left(\frac{v}{c}\right)^{k-1} \exp \left[-\left(-\frac{v}{c}\right)^{k}\right]
$$

In the equation: $k$ represents shape factor; $C$ represents the average wind speed as described area, $v$ represents for wind farm wind speed, the unit is $\mathrm{m} / \mathrm{s}$. Let the wind farm output can be used (2) to obtain:

$$
p_{W}=\left\{\begin{array}{lc}
0 & v<v_{\text {in }} n v>v_{\text {out }} \\
\frac{v-v_{\text {in }}}{v_{r}-v_{\text {in }}} P_{W N} & v_{\text {in }} £ v<v_{r} \\
P_{W N} & v_{r} £ v £ v_{\text {out }}
\end{array}\right.
$$

In the equation: $p_{\mathrm{w}}, P_{\mathrm{wN}}, v, v_{\mathrm{in}}, v_{\mathrm{r}}, v_{\text {out }}$ denote wind farm output, rated output, the actual wind speed, cut-in speed, rated wind speed and cut-out wind speed respectively.

According to formula (1) and (2) paper inferred that the probability density function of the wind farm wind power output is:

$$
\left\{\begin{array}{l}
\phi\left(p_{\mathrm{W}}\right)=\frac{k\left(v_{\mathrm{r}}-v_{\text {in }}\right)}{P_{\mathrm{WN}} c}\left[\frac{p_{\mathrm{W}}\left(v_{\mathrm{r}}-v_{\text {in }}\right)+P_{\mathrm{WN}} v_{\text {in }}}{P_{\mathrm{WN}} c}\right]^{k-1} \exp \left\{-\left[\frac{p_{\mathrm{W}}\left(v_{\mathrm{r}}-v_{\text {in }}\right)+P_{\mathrm{WN}} v_{\text {in }}}{P_{\mathrm{WN}} c}\right]^{k}\right\} \quad v_{\text {in }} \leq v<v_{\mathrm{r}} \\
P\left(p_{\mathrm{W}}=0\right)=\int_{0}^{v_{\text {in }}} \phi(v) \mathrm{d} v+\int_{v_{\text {out }}}^{+\infty} \phi(v) \mathrm{d} v=1-\exp \left[-\left(\frac{v_{\text {in }}}{c}\right)^{k}\right]+\exp \left[-\left(-\frac{v_{\text {out }}}{c}\right)^{k}\right] \quad v<v_{\text {in }} \text { or } v>v_{\text {out }} \\
P\left(p_{\mathrm{W}}=P_{\mathrm{WN}}\right)=\int_{v_{\mathrm{r}}}^{v_{\text {out }}} \phi(v) \mathrm{d} v=\exp \left[-\left(\frac{v_{\mathrm{r}}}{c}\right)^{k}\right]-\exp \left[-\left(\frac{v_{\text {out }}}{c}\right)^{k}\right] \quad v_{\mathrm{r}} \leq v \leq v_{\text {out }}
\end{array}\right.
$$

\section{Wind power business participation market description}

Day-ahead market formulates the next day unit combination regimen and power distribution plan. Due to the weather, grid failure, sudden load change and other factors, real-time market are brought about day-ahead market and actual load appears a large deviation. In the day-ahead market, according to short-term wind forecasting results or other bidding strategy, wind farm business declares output and price. Currently, to energy conservation, power grid companies purchase all the wind power, therefore and it can be assumed that wind power business price quotation is zero on the day-ahead market, that wind electricity business is recipients of market clearing price today. It can be supposed that power grid companies adopt unified clearing price settlement and power suppliers clearing in the day-ahead market, including wind farm business.

\section{Wind farm business day-ahead market bidding strategy}

When the deviation $\left|P_{\mathrm{w}, t}-p_{\mathrm{w}, t}\right|$ of between wind farm businesses declaration output $P_{\mathrm{w}, t}$ in 
period $t$ and the actual output of the wind farm $P_{\mathrm{w}, t}$ less than the allowable deviation $\alpha^{*} P_{\mathrm{w}, t}$, wind farm business gains clearing in accordance with the size of the actual output proceed to checkout in day-ahead market, there is not imbalance clearing costs; when the deviation $\left|P_{\mathrm{w}, t}-p_{\mathrm{w}, t}\right|$ is greater than $\alpha^{*} P_{\mathrm{w}, t}$, in day-ahead market the output of wind farm in accordance with the output of the wind farm businesses declaration and allowable deviation proceed to checkout, imbalance power of other exceed allowable deviation is settled in the imbalance market. Because it contains the wind power random variable, therefore, the objective function is a desired value:

$$
E(R)=\max E\left(\sum_{t=1}^{N}\left(X_{t} \rho_{t}+N B_{\mathrm{up}, t}-N B_{\text {under }, t}\right)\right)
$$

In the formula, $E(R)$ represents the wind farm business expect earnings; $N$ represents the number of hours day-ahead market $N$ refers to the number of periods a day, usually $24 \mathrm{~h}$, so the formula is omitted in time; $N B_{\text {up }, t}$ said that due to the day-ahead market declare output less, more than the allowable deviation, excess output in the imbalance market to sell obtain earnings; $N B_{\text {under, } t}$ said that due to the day-ahead market declare output more than the allowable deviation, the loss of revenue of lack of power in the unbalanced market purchasing; $\rho_{t}$ represents day-ahead market $t$ hours market clearing price; $X_{t}$ represents variable that wind farm businesses in the day-ahead market each period before declaration output and tolerance output after comprehensive consideration.

In the formula (4), the first part represents benefits of wind farm businesses in the day-ahead market declaration output to obtain, $X_{t}$ can be expressed as follows:

$$
X_{t}= \begin{cases}P_{\mathrm{w}, t} & \left|P_{\mathrm{w}, t}-p_{\mathrm{w}, t}\right| \leq \alpha^{*} P_{\mathrm{w}, t} \\ P_{\mathrm{w}, t}+\alpha^{*} P_{\mathrm{w}, t} & p_{\mathrm{w}, t}-P_{\mathrm{w}, t}>\alpha^{*} P_{\mathrm{w}, t} \\ P_{\mathrm{w}, t}-\alpha^{*} P_{\mathrm{w}, t} & P_{\mathrm{w}, t}-P_{\mathrm{w}, t}>\alpha^{*} P_{\mathrm{w}, t}\end{cases}
$$

In the formula (5), $P_{\mathrm{w}, t}$ is the wind farm market businesses declaration output in the day-ahead market $t$ period, represents the decision variables; $\alpha$ is allowed the wind power output deviation proportion.

In the formula (4), contribute to the second part $N B_{\text {up }, t}$ represents the obtained revenue by selling extra output in the imbalance market, namely:

$$
N B_{\mathrm{up}, t}= \begin{cases}\rho_{\mathrm{sell}, t} \int_{(1+\alpha) P_{\mathrm{ww}, t}}^{P_{\mathrm{w}}}\left(\left(p_{\mathrm{w}, t}-(1+\alpha) P_{\mathrm{w}, t}\right) \phi\left(p_{\mathrm{w}, t}\right) \mathrm{d} p_{\mathrm{w}, t}\right. & I_{\mathrm{up}, t}>0 \\ 0 & I_{\mathrm{up}, t} \leq 0\end{cases}
$$

In the formula (6), $\rho_{\text {sell,t }}$ represents price of wind farm businesses sell excess power in imbalance market.

When $I_{\mathrm{up}, t}>0$, the formula (6) can be further converted to:

$$
\begin{gathered}
N B_{\text {up }, t}=\rho_{\text {sell }, t} * \frac{P_{W N} c}{\left(v_{r}-v_{\text {in }}\right)}\left\{\Gamma\left(\frac{1}{k}+1\right)-\gamma\left[\frac{1}{k}+1,\left(a+\frac{v_{\text {in }}}{c}\right)^{k}\right]-\Gamma\left[\frac{1}{k}+1,\left(b+\frac{v_{\text {in }}}{c}\right)^{k}\right]\right\} \\
-\rho_{\text {sell }, t} *\left(\frac{P_{W N} c}{\left(v_{r}-v_{\text {in }}\right)} \frac{v_{\text {in }}}{c}+(1+\alpha) P_{w, t}\right)\left\{\exp \left[-\left(a+\frac{v_{\text {in }}}{c}\right)^{k}\right]-\exp \left[-\left(b+\frac{v_{\text {in }}}{c}\right)^{k}\right]\right\}
\end{gathered}
$$

Among them, $\Gamma\left(\frac{1}{k}+1\right), \gamma\left[\frac{1}{k}+1,\left(a+\frac{\left.v_{\text {in }}\right)^{k}}{c}\right], \Gamma\left[\frac{1}{k}+1,\left(b+\frac{v_{\text {in }}}{c}\right)^{k}\right]\right.$, they respectively are gamma function, incomplete gamma function, upper incomplete gamma function; $a=\frac{(1+\alpha) P_{\mathrm{w}, t}\left(v_{\mathrm{r}}-v_{\mathrm{in}}\right)}{P_{\mathrm{WN}}}, b=\frac{v_{\mathrm{r}}-v_{\text {in }}}{c}$

In the formula (4), where the third part $N B_{\text {under, } t}$ represents the loss of income that wind farm businesses buy a lack of power in the imbalance market, namely:

$N B_{\text {under }, t}= \begin{cases}\rho_{\text {buy, }, t} \int_{0}^{(1-\alpha) p_{\mathrm{w}, t}}\left((1-\alpha) P_{\mathrm{w}, t}-p_{\mathrm{w}, t}\right) \rho\left(p_{\mathrm{w}, t}\right) \mathrm{d} p_{\mathrm{w}, t} & I_{\mathrm{dow}, t}>0 \\ 0 & I_{\text {down }, t} \leq 0\end{cases}$

In the formula $(8), \quad I_{\text {dow }, t}=\max \left(\left((1-\alpha)^{*} P_{\mathrm{w}, t}-p_{\mathrm{w}, t}\right), 0\right) ; \quad \rho_{\text {buy }, t}$ represents price that market wind farm 
businesses purchase less power in the imbalance market.

When $I_{\text {down }, t}>0$, the equation can be further converted to:

$$
\begin{aligned}
& N B_{\text {under }}=\rho_{\text {buy }, t} *\left((1-\alpha) P_{\mathrm{w}, t}+\frac{P_{\mathrm{wN}} c}{\left(v_{\mathrm{r}}-v_{\text {in }}\right)} \frac{v_{\text {in }}}{c}\right)\left\{\operatorname { e x p } \left[-\left(\frac{v_{\text {in }}}{c}\right)^{\mathrm{k}}-\exp \left[-\left(f+\frac{v_{\text {in }}}{c}\right)^{\mathrm{k}}\right\}\right.\right. \\
& -\rho_{\text {buy }, t} * \frac{P_{\mathrm{wN}} c}{\left(v_{\mathrm{r}}-v_{\text {in }}\right)}\left\{\Gamma\left(\frac{1}{k}+1\right)-\gamma\left[\frac{1}{k}+1,\left(\frac{v_{\text {in }}}{c}\right)^{k}\right]-\Gamma\left[\frac{1}{k}+1,\left(f+\frac{v_{\text {in }}}{c}\right)^{k}\right]\right\}
\end{aligned}
$$

Among them, $f=\frac{(1-\alpha) P_{\mathrm{w}, t}\left(v_{\mathrm{r}}-v_{\mathrm{in}}\right)}{P_{\mathrm{wN}} c}$.

In the day-ahead market, wind farm businesses do not know the actual output, actual output is a random variable, so it can only be a desired value.

$$
\bar{p}_{w, t}=\int_{0}^{\infty} p_{\mathrm{w}, \mathrm{t}} \phi\left(p_{\mathrm{w}, \mathrm{t}}\right) \mathrm{d} p_{\mathrm{w}, t}=\frac{P_{\mathrm{wN}} c}{\left(v_{\mathrm{r}}-v_{\mathrm{in}}\right)} \Gamma\left(\frac{1}{k}+1\right)-\frac{P_{\mathrm{wN}} c}{\left(v_{\mathrm{r}}-v_{\mathrm{in}}\right)} \frac{v_{\mathrm{in}}}{c} \exp \left[-\left(\frac{v_{\mathrm{in}}}{c}\right)^{k}\right]
$$

In order to promote wind power businesses provide power as accurately as possible in the day-ahead market, in general, the price is set to $\rho_{\text {buy }, t} \geq \rho_{t} \geq \rho_{\text {sell, },} \geq 0$.

Since the distribution of electricity is difficult to use a specific distribution function to describe, load levels and network structure are different, distribution has a difference, so this paper directly adopts the result of the day-ahead market price predicted as the decision basis of wind farm businesses declaration output.

\section{Operator case study}

A wind farm parameters: $P_{\mathrm{wN}}=50 \mathrm{MW}, v_{\mathrm{in}}=3 \mathrm{~m} \mathrm{~s}, v_{\mathrm{r}}=15 \mathrm{~m} / \mathrm{s}, \quad v_{\text {out }}=25 \mathrm{~m} / \mathrm{s}$. it is supposed that $\rho_{\text {sell,t }}=0.6 \rho_{t} \quad \rho_{\text {buy }, t}=1.4 \rho_{t}$ in the imbalance market. The wind energy prediction data is satisfied with the Weibull distribution, the use of maximum likelihood method to get the Weibull distribution parameters is $k=1.7627, c=6.2609$. For example, in the 0 period, short-term forecasting output value is $10.25 \mathrm{MW}$, the predicted clearing price is $\$ 44.59 / \mathrm{MWh}$, wind power output mean is 13.7142MW.

(1)The influences of permissible deviation ratio for the day-ahead market declared output and income

As can be seen from Figure 1, as allowable proportion deviation is gradual increasing, the wind farm businesses bidding output on the day-ahead market from the $13.7142 \mathrm{MW}$ gradually increased 17.0363MW ( $C=6.2609$ ). At this time, it is unnecessary for wind farm businesses to bear the cost of imbalance, because the allowable deviation is $\alpha$, in order to obtain the maximum benefits, the wind farm businesses will increase bidding output to the best bidding output in the day-ahead market, that is $(1+\alpha) \bar{p}_{w, t}$, Instead of the predicted value $10.25 \mathrm{MW}$. Visible, as long as clearing price and the imbalance market price to meet $\rho_{\text {buy }, t} \geq \rho_{t} \geq \rho_{\text {sell,t }} \geq 0$ in the day-ahead market, the above bidding strategy model is able to promote power suppliers to declare output as much as possible in accordance with wind resources, reduce the imbalance power of wind power fluctuations resulting in, optimize resource allocation, improve system reliability and power suppliers earnings. As can be seen from Figure 1 and Figure 2, with $c$ value is increasing in the Weibull parameters, the wind farm businesses best bidding output is increasing in day-ahead market, the corresponding wind farm businesses income is also increasing.

As can be seen from Figure 3, as allowable deviation proportion is gradual increasing, the wind farm businesses bidding output on the day-ahead market from the $12.0975 \mathrm{MW}$ gradually increased 15.0279MW $(k=3)$, the same equal $(1+\alpha) \bar{p}_{\mathrm{w}, t}$. As can be seen from Figures 3 and 4 , with $k$ value is increasing in the Weibull parameter, the wind farm businesses reduce the day-ahead market best bidding output, correspondingly earnings are lowering.

(2)Under the same allowable deviation, the influence of $k$ and $C$ for declaration output

Figures 5 and 6 respectively reflect with increasing of $k$ and $C$, the wind farm businesses 
bidding output is also changing on the day-ahead market. As $k$ value increases, in order to make desirable earnings maximize, bidding output is decreasing, because the desired output is reduced.

With the increase of $C$ value, the best bidding output is gradually increasing, until the rated power. Because of the $C$ increase represents an increase of wind farm wind resource, so the actual wind power output is certainly increasing, the output of declaration is corresponding increasing.

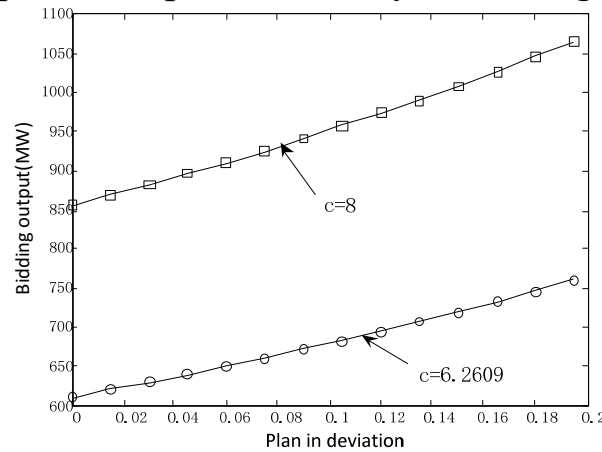

Fig 1 changing of bidding power with allowable deviation and $c$

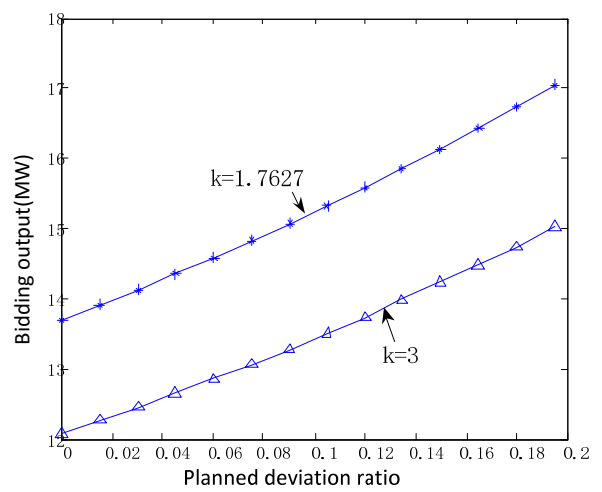

Fig 3 changing of bidding power with allowable deviation and $k$

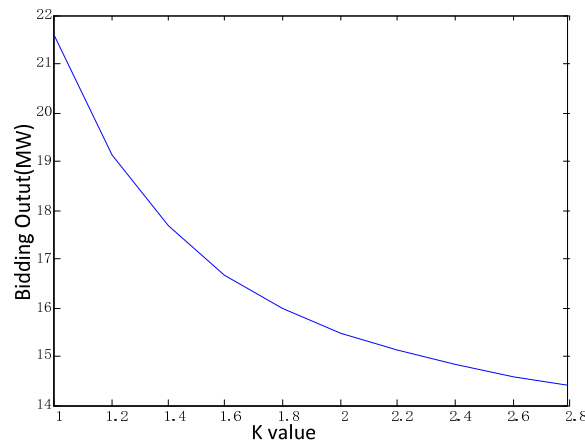

Fig 5 changing of bidding power with $k$

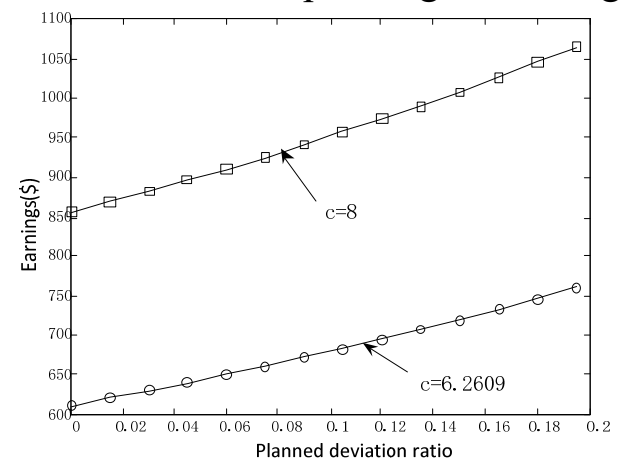

Fig 2 changing of bidding revenue with allowable deviation and $c$

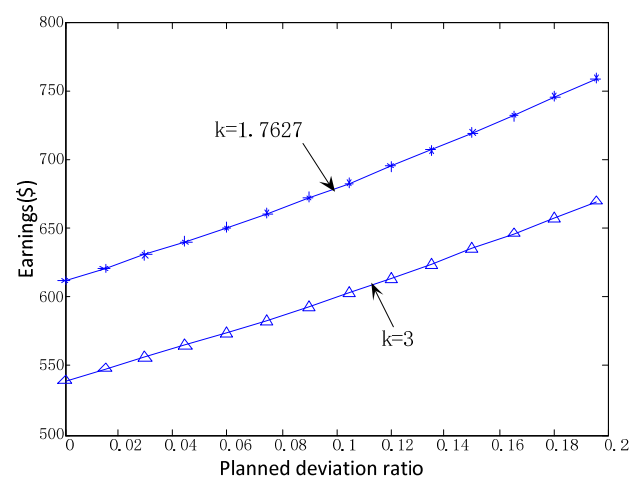

Fig 4 changing of bidding revenue with allowable deviation and $k$

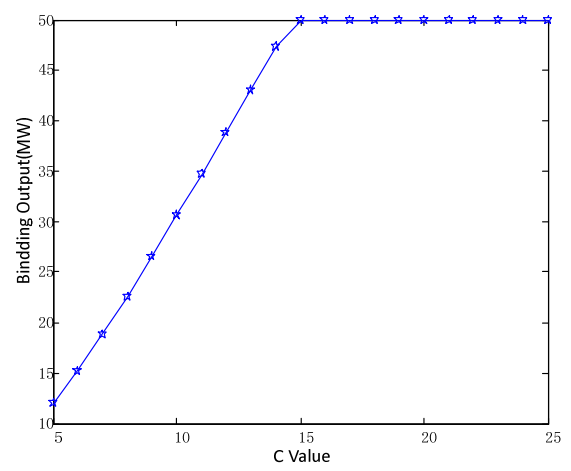

Fig 6 changing of bidding power with $C$

\section{Conclusions}

With the large-scale development of wind power, wind power participates in market transactions what is an inevitable trend. Because of the volatility of wind power and the feature of inaccurately predict, wind power can't be exactly the same as the conventional energy market to participate in competing. This paper put forward to consider wind power fluctuation allowable deviation bidding strategy model, and analyzed examples in detail, specific conclusions are as follows:

(1) The wind power involved in market bidding, it is necessary to appropriate loosen the accuracy of its declaration output, namely allowable deviation, the deviation level may be adjusted according to factors such as wind speed forecasting accuracy; 
(2) With the increase of allowed deviation proportion levels, regardless of how the wind speed distribution parameters $k$ and $C$ values value, the wind farm's optimal output bids are increasing, earnings also follow the increase;

(3) Under the same allowable deviation levels, the increase of wind speed distribution parameter values will reduce the wind power businesses income, bidding output is also gradually decreasing. On the contrary, the increase of wind speed distribution parameter $C$ value will improve wind power businesses income, bidding output is also gradually improving until the rated output.

\section{References}

[1] Global Wind Energy Council. 2013 Global wind electric statistics. Wind Energy, 2014.

[2] China Wind Energy Association. 2013 China wind electric installed capacity statistical. Wind Energy, 2014 (2) 44-45.

[3] Wu Zhengqiu, Wang Tao.Deviation management of wind power prediction and decision-making of wind power bidding.Power System Technology, 2011 (12) 160-163.

[4] Jiaqi Liang, Grijalva, S. Harley, R.G. Increased Wind Revenue and System Security by Trading Wind Power in Energy and Regulation Reserve Markets. IEEE Transactions on Sustainable Energy, 2011 (2) 340-347.

[5] Angarita,J.L.,Hernandez,C.A.,Usaola,J..Analysis of a wind farm's revenue in the British and Spanish markets[J]. Energy Policy, 2007 (10) 5051 - 5059.

[6] U saola, J.; Moreno, M.A..Optimal Bidding of Wind Energy in Intraday Markets.Energy Market[C], 6th EEM International Conference, European, 2009 (2) 1-7. 